\title{
Polymer Optical Fiber Compound Parabolic Concentrator fiber tip based glucose sensor: In-Vitro Testing
}

\author{
Hassan, Hafeez UI; Janting, Jakob; Aasmul, Soren; Bang, Ole
}

Published in:

IEEE Sensors Journal

Link to article, DOI:

10.1109/JSEN.2016.2606580

Publication date:

2016

Document Version

Peer reviewed version

Link back to DTU Orbit

Citation (APA):

Hassan, H. U., Janting, J., Aasmul, S., \& Bang, O. (2016). Polymer Optical Fiber Compound Parabolic Concentrator fiber tip based glucose sensor: In-Vitro Testing. IEEE Sensors Journal, 16(23), 8483 - 8488. [7562488]. https://doi.org/10.1109/JSEN.2016.2606580

\section{General rights}

Copyright and moral rights for the publications made accessible in the public portal are retained by the authors and/or other copyright owners and it is a condition of accessing publications that users recognise and abide by the legal requirements associated with these rights.

- Users may download and print one copy of any publication from the public portal for the purpose of private study or research.

- You may not further distribute the material or use it for any profit-making activity or commercial gain

- You may freely distribute the URL identifying the publication in the public portal 


\title{
Polymer Optical Fiber Compound Parabolic Concentrator fiber tip based glucose sensor: in-Vitro Testing
}

\author{
Hafeez Ul Hassan, Jakob Janting, Soren Aasmul, and Ole Bang
}

\begin{abstract}
We present in-vitro sensing of glucose using a newly developed efficient optical fiber glucose sensor based on a Compound Parabolic Concentrator (CPC) tipped polymer optical fiber (POF). A batch of 9 CPC tipped POF sensors with a $35 \mathrm{~mm}$ fiber length is shown to have an enhanced fluorescence pickup efficiency with an average increment factor of 1.7 as compared to standard POF sensors with a plane cut fiber tip. Invitro measurements for two glucose concentrations (40 and 400 $\mathrm{mg} / \mathrm{dL}$ ) confirm that the CPC tipped sensors efficiently can detect both glucose concentrations.
\end{abstract}

Index Terms - Compound Parabolic Concentrator, Fiber optics sensors, Fluorescence, Polymers.

\section{INTRODUCTION}

According to the World Health Organization, diabetes will be the 7th leading cause of death in 2030 [1]. Diabetes is a disease that prevents patients from controlling the glucose level and, if untreated or improperly managed, leads to a variety of complications, such as a slow damage of both the small and large vessels in the body [2]. Both a too high (hyperglycemia) and a too low (hypoglycemia) glucose level over extended periods can cause chronic complications [3]. Insulin is a hormone that regulates the blood glucose levels and proper insulin delivery based on continuous glucose monitoring is extremely important for diabetes patients. This requires a low-cost, accurate (as defined clinically by error grid analysis [4]) and bio-compatible sensor, which is easy to operate and can be placed in the patient body for frequent or continuous glucose measurements.

Recently the interest in fluorescence based fiber-optical sensors has increased, as they can be used for measuring

This paragraph of the first footnote will contain the date on which you submitted your paper for review. This work was funded by the People Programme (Marie Curie Actions) of the European Union's Seventh Framework Programme FP7/2007-2013/ under REA grant agreement

Hu. Hassan, J. Janting and O. Bang are with DTU Fotonik, Department of Photonics Engineering, Technical University of Denmark, DK-2800 Kgs. Lyngby, Denmark (e-mail: hafha@fotonik.dtu.dk; jajant@fotonik.dtu.dk; oban@fotonik.dtu.dk).

S. Aasmul is with Medtronic Research and Technology Bakken Research Center BV, Endepolsdomein 5, 6229 GW Maastricht, Netherlands (email: soren.aasmul@medtronic.com). various physical and biological quantities, such as aluminum ions [5], glucose [3,6,7], $\alpha$-streptavidin and $\alpha$-CRP antibodies [8-10], and salmonella bacteria [11]. A special type of these sensors uses so-called FRET (Förster Resonance energy transfer) as the sensing mechanism $[3,6,7,11,12]$. FRET is an intermolecular phenomenon of radiation less energy transfer between an excited donor fluorophore and a proximal ground state acceptor (a fluorophore or a dye) through a long range dipole-dipole interaction [13] and it can be used for the measurement of intermolecular distances in the range of Ångstrøms (10-100 $)$ [14]. Two main fiber-based FRET sensor configurations exist, which is binding the FRET pair to the surface of the fiber and use evanescent-wave excitation [11] or bonding a compartment to the fiber tip, which contains the FRET pair in solution $[3,6,15]$.

FRET based fiber-optical biosensors for continuous glucose monitoring have been proposed by several groups $[3,6,7]$ as an alternative to the electrochemical sensors [16,17]. Compared to electrochemical sensors, which consume glucose for sensing, the FRET based sensors are affinity based and equilibrium driven, which results in their sensitivity being independent on the rate of glucose diffusion [6]. In addition they do not produce auto-destructive side products, such as hydrogen peroxide, which is a problem in for example first generation electrochemical sensors, where glucose is measured by hydrogen peroxide quantification based on its electrochemical oxidation on metal based electrodes [18-20].

One limitation for FRET based sensors in general is, however, that they suffer from a low signal to noise ratio [21], which can affect their accuracy and the lower limit of detectable analyte concentration. For evanescent-wave sensing the overlap between the excitation light and FRET pairs can be increased by tapering the fiber tip to increase the evanescent light [11], but that does necessarily increase the pick-up efficiency [22] and thereby the signal-to-noise ratio. For the FRET-based fiber-optical sensors using a compartment bonded to the end of the fiber, the problem can be overcome by increasing the numerical aperture of the fiber tip to improve both the excitation and the fluorescence pickup efficiency. One way to do this is to shape the fiber tip as a compound parabolic concentrator (CPC). The CPC is widely 
used in solar energy systems and many other applications where concentration of light from a highly divergent source is required, and was recently demonstrated to be applicable also for fluorescence based POF biosensors [15]. The CPC geometry in 2 dimensions is shown in Figure 1(a) and its more detailed description can be found in the literature [23]. The CPC fiber tip conserves the etendue [23], which means that the numerical aperture at the output is increased due to the reduced output aperture. As the fluorescence is emitted isotopically, increasing the numerical aperture of the fiber tip, will allow it to capture more light compared to the plane cut fiber tip.

(a)

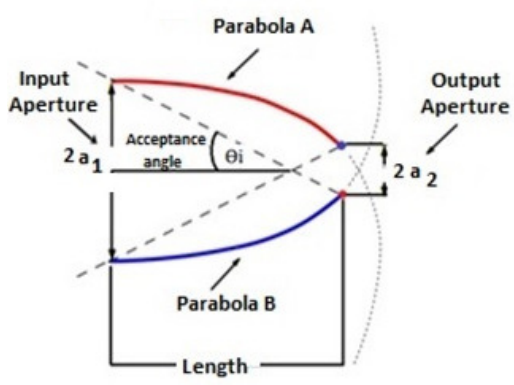

(b)

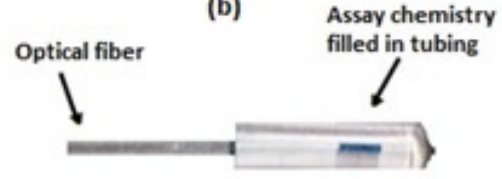

(c)

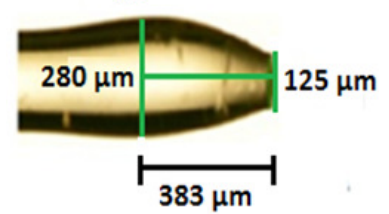

Fig. 1. (a) Compound Parabolic Concentrator geometry with input aperture $2 \mathrm{a}_{1}$, acceptance angle $\Theta_{\mathrm{i}}$ and output aperture $2 \mathrm{a}_{2}$. (b) Dummy sensor (c) Microscopic image of the produced CPC-tip showing an increase in diameter at the beginning of the $\mathrm{CPC}$ shape.

Both silica optical fibers [3] and polymer optical fibers (POFs) [6] have been used for FRET-based glucose sensing, but POFs have several advantages such as an inherent biocompatibility and good flexibility [24], where broken silica fibers obviously would be hazardous for the body. Neither FRET-based silica fiber nor POF glucose sensors have been optimized by tip shaping.

In our previous work [15], we made CPC tipped POF sensors and evaluated their fluorescence light pick-up efficiency by using a dummy sensor configuration [see Figure 1(b)], in which they sensed the fluorescence of a real glucose assay in a tube, which is not glucose permeable. These initial results confirmed that $\mathrm{CPC}$ fiber tips can indeed increase the fluorescence signal pick-up efficiency by a factor of 1.7 compared to a standard plane-cut fiber tip, even using a nonoptimal fiber length. However, it is important to verify that the improved CPC tipped POFs can be used in a real glucose sensor by in-vitro experiments. Several complications make this non-trivial, e.g., can the assay containing membrane be properly bonded to a non-uniform fiber tip with a localized increase in the fiber diameter [see Figure 1(c)] and how well can the assay concentration be controlled in the sensor manufacturing process? In this paper we report the results of such in-vitro glucose sensor experiments.

\section{MATERIALS AND METHODS}

\section{A. Glucose Sensor}

The glucose sensor consists of a POF bonded to a glucose permeable membrane to make a compartment in which the fluorescence assay chemistry is filled. The permeable membrane allows the diffusion of glucose molecules into the chemistry when placed in a glucose solution. The glucose sensor and assay chemistry are shown in Figure 2(a) and 2(b), respectively. The assay consists of a glucose binding protein and a glucose analog (green chain) labelled with a fluorophore (Alexa fluor 594: $\lambda$ excitation $=590 \mathrm{~nm}, \lambda$ emission $=618 \mathrm{~nm}$ ) (orange dots) and a crystal violet dye (blue dots), respectively, which make a FRET pair. The glucose analog competes with the glucose on binding to the protein and eventually the system reaches an equilibrium, which correlates to the glucose concentration. The equilibrium state of the assay is optically interrogated by measuring the intensity of the emitted fluorescence. Low glucose concentration results in close proximity of the glucose binding protein (labelled with a fluorophore) and the glucose analog (labelled with a dye), which will lead to a high degree of FRET between the labelled fluorophore and dye and thus a lower fluorescence intensity. High concentration of the glucose in the assay will result in low FRET, which will lead to high fluorescence intensity, as shown in Figure 2(c).

The measured intensity of the emitted fluorescence will depend on the intensity of the light source and the coupling between the assay and the optical system. A reference fluorophore (Alexa fluor 700: $\lambda$ excitation $=633-647 \mathrm{~nm}, \lambda$ emission $=723 \mathrm{~nm}$ ), which is unaffected by the glucose concentration, is therefore included in the assay to eliminate any unwanted fluctuations due to this dependency. The ratio between the assay and reference fluorescence is used to determine the glucose concentration. 


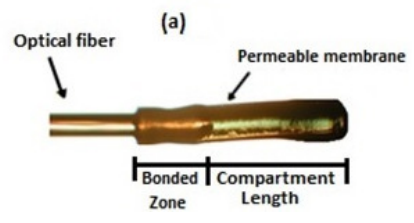

(b)

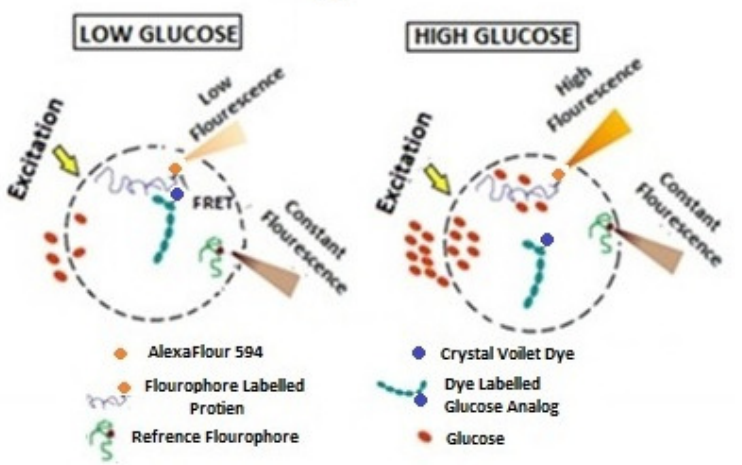

(c)

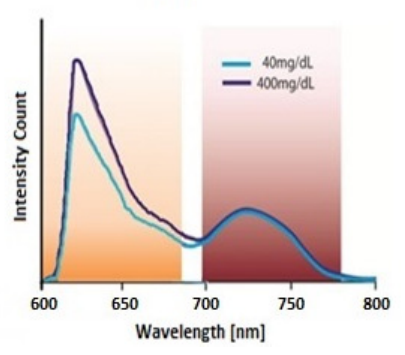

Fig. 2. (a) Real glucose sensor; (b) Illustration of the assay chemistry and FRET sensing principle resulting in (c) low and high intensity for $(40 \mathrm{mg} / \mathrm{dL})$ and $(400 \mathrm{mg} / \mathrm{dL})$ respectively.

\section{B. Optical Setup}

The sensors are characterized using an epi-fluorescence setup and a fiber optical spectrometer (USB2000+, Ocean Optics, FL, USA), as shown in Figure 3. The setup consists of a light source (LED) HLMP-EL30-MQ000 with center wavelength of $590 \mathrm{~nm}$ that passes through a $55 \mathrm{~nm}$ excitation filter with $560 \mathrm{~nm}$ central wavelength and a beam splitter and is coupled to the fiber by a lens to excite the assay chemistry. The resulting fluorescence from the assay is picked up by the fiber and passed through a beam splitter and a long-pass emission filter with $610 \mathrm{~nm}$ cut-off wavelength to finally reach the spectrometer.

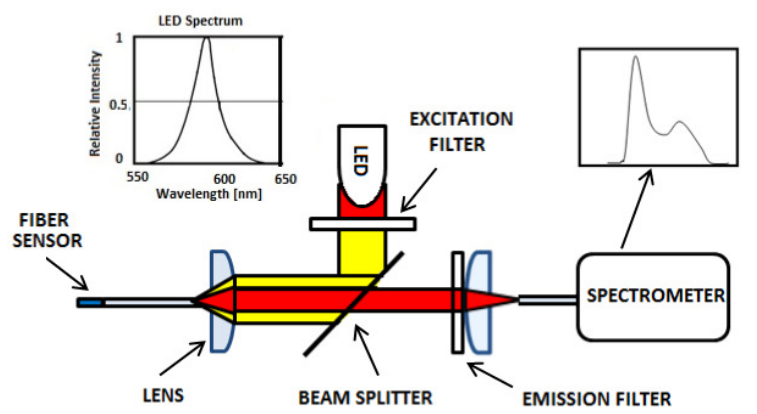

Fig. 3. Optical Setup for sensor characterization.

\section{Compound Parabolic Concentrator}

The POF was a commercial medical grade Super ESKA PMMA fiber with a $240 \mu \mathrm{m}$ core diameter and a $250 \mu \mathrm{m}$ outer diameter. The complete CPC manufacturing process is explained in our previous work [15], in which the specifications of the ideal CPC shape for a POF glucose sensor were studied. For a total POF length of $35 \mathrm{~mm}$ and taking into account restrictions in the CPC fabrication, a CPC with an output fiber diameter of $125 \mu \mathrm{m}$ and a CPC length of $311 \mu \mathrm{m}$ was chosen, for which a factor of 3 improvement in pick-up efficiency as compared to the plane-cut fiber tipped sensor, was predicted. An average improvement factor of 1.7 was found experimentally using the dummy sensor configuration described above [15].

In this paper, a new batch of 9 CPCs has been made by parabolic tapering of the fiber at $190^{\circ} \mathrm{C}$, using the heat and pull method. The resulting tapers were then cut and polished to the above specified diameter to make a CPC tip as already shown in Figure 1(c). The CPC tips manufactured by this method were not completely uniform in shape. A general feature for example is that at the start of a CPC, the diameter increases to a certain maximum diameter before decreasing monotonically to the chosen diameter, as previously shown in Figure 1(c). Furthermore, the CPCs have a longer length than the ideal CPC length. While the fabricated CPCs have a nonideal shape, the shape they do have is maintained for all 9 CPCs with a low standard deviation of the maximum diameter and the length.

\section{Sensor Fabrication and Characterization}

Each CPC-tipped fiber was first optically characterized three times for its increment factor in a dummy sensor configuration using the optical setup described above. In between each measurement with the same CPC-tipped fiber it was cleaned and inserted in a new cuvette with a fresh assay from the same volume of chemistry. The three repetitions provide a reasonable statistical average and therefore reduce the effect of coupling variations.

After characterization in the dummy sensor configuration, each fiber was cleaned and used to make a real glucose sensor, where a glucose permeable membrane of $65 \mu \mathrm{m}$ thickness with a $5 \mathrm{~mm}$ length and $250 \mu \mathrm{m}$ inner diameter, was bonded to the fiber to make an assay compartment. The bonding takes up a certain length, so that the final compartment length was about $2.05 \mathrm{~mm}$, as seen in Figure 2(a). Using a syringe with a 34 Gauge needle with an inner/outer diameter of $83 / 184 \mu \mathrm{m}$ the compartment was then partly filled with a fixed volume of $50 \mathrm{~nL}$ of the assay and sealed from the other end. The reason to use $5 \mathrm{~mm}$ rather long membranes is to have efficient filling and sealing. The sensors were then pressurized in a buffer solution of $\mathrm{pH} 7.02$ for 24 hours. Pressurization was done by closing the tip of syringe (inner diameter $15.6 \mathrm{~mm}$ ) by melting, 
filling it half with the buffer solution containing the sensor, and pressing the piston in to half the air volume, giving ideally a pressure of 2 bars. Putting the sensors in the buffer solution diluted the assay, as the buffer solution enters the compartment through the membrane and therefore fills it completely. Pressurization is required to remove any air bubbles inside the compartment during dilution of the assay. For performance comparison, two sensors have also been made using plane-cut fiber tips.

The real sensors were then characterized for their increment factor using the same optical setup as for the dummy sensor characterization, shown in Figure 3. The increment factor is here defined as the ratio of the detected intensity of the real CPC-tipped sensor and the real plane-cut sensor at $618 \mathrm{~nm}$.

The CPC-tipped real glucose sensors [Figure 2(a)] were then characterized for their glucose response. For glucose measurements, the sensors were placed in a buffer solution of two known glucose concentrations of $40 \mathrm{mg} / \mathrm{dL}$ and 400 $\mathrm{mg} / \mathrm{dL}$ at $37^{\circ} \mathrm{C}$, to emulate the human body temperature in an in-vitro experiment. The output intensity spectrum of the sensors is measured first for the low glucose concentration at different times of 20, 40 and 60 minutes. The spectrum remained the same for all measurements, which indicates that the sensor reached equilibrium in 20 mins or less. The same procedure is then repeated for high glucose concentration.

It should be noted that the response time of the sensor can be further reduced by reducing the glucose permeable compartment wall thickness and thus allowing the faster diffusion of the glucose into the assay.

\section{RESULTS}

The recorded spectrum for the real sensors when characterized in no glucose concentration is shown in Figure 4(a). Figure 4(b) shows the increment factor for both dummy and real sensors. Figure 4(d) shows the fluorescence spectrum of a sensor with a plane cut tip and of two selected CPCtipped sensors at different glucose concentrations.

It is interesting to note from Figure 4(b) that the increment factor for some of the real sensors deviates from that found from the dummy sensors. Ideally the increment factor should be the same for the real and dummy sensors. From Figure 4(b), we note that the real CPC 6 sensor showed no increment. A close analysis of this sensor showed that there were bubbles in front of the CPC tip, as shown in Figure 4(c), which means that they were not completely dissolved in the assay during pressurization. This results in a low detected intensity because of the reduced amount of assay for interrogation in front of the fiber tip. This sensor is therefore discarded in the further analysis.

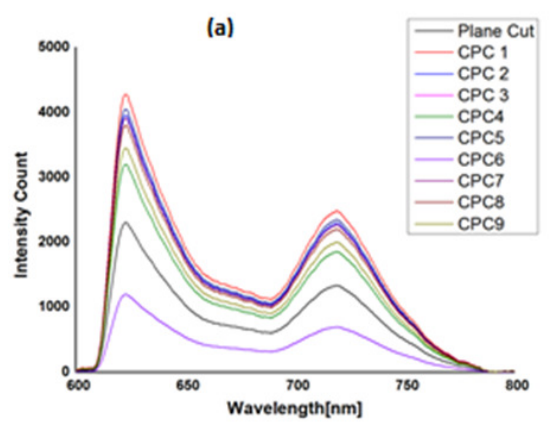

(b)

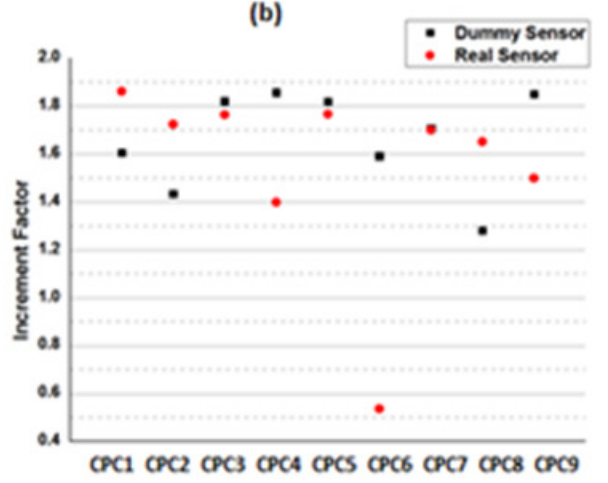

(c)
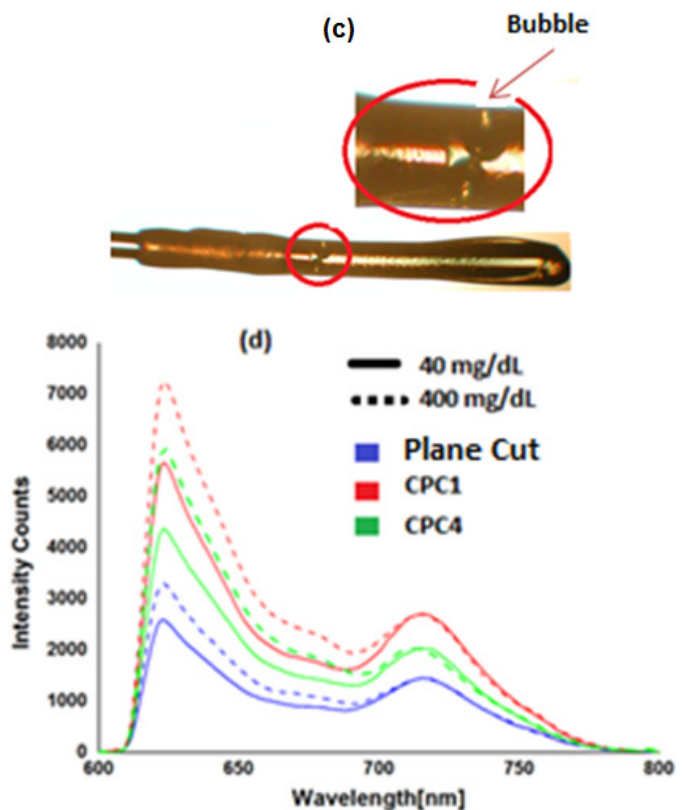

Fig. 4. (a) Fluorescence spectrum for CPC-tipped and plane-cut real sensors without glucose; (b) Increment factor for both dummy and real CPC based sensors; (c) Bubbles in the assay for CPC 6; (d) Fluorescence spectrum for Plane Cut and two selected CPC based real sensors at different glucose concentrations.

As mentioned in our previous work, because of the variation in the maximum diameter and the CPC length due to fabrication process limitations, the maximum increment factor of 3 is not achieved and the increment factor for the CPCs varies. The average increment factor for the complete batch of CPC-tipped sensors (the real CPC 6 sensor omitted) is 1.7 for both the real and the dummy sensors, separately.

We evaluate that the deviation in the increment factor between real and dummy sensors is primarily due to the 
difference in the overall assay concentration. Even though the sensors are initially filled with the same volume of assay the final concentration of the assay, after pressurization and dilution, may vary due to a difference in compartment length of the sensors. One reason for the variation in compartment length is due to the manual process of sliding the membrane onto the fiber for bonding, which makes it difficult to keep a constant length of the bonded zone (see Figure 2(a)).

To study the correlation between the increment factor and the compartment length we made detailed measurements of the compartment length of all 8 sensors and calculated the two-parameter Pearson's correlation coefficient between them to be 0.7 . This shows a strong and positive correlation, given that the assay volume and the plane-cut sensor used for the increment factor calculations are fixed, which can explain the deviation in the increment factor compared to the dummy sensors.

\section{DISCUSSION}

In our earlier work [15] we demonstrated that limitations in the current tapering fabrication process of the CPC were the reason behind fluctuations in the CPC geometry and thereby the reason for not achieving the predicted ideal increment factor of 3.0. There is thus a clear route towards achieving the optimum CPC shape and the optimum performance of the CPC tipped sensor by improving the fabrication process.

Our results here have shown that the increment factor of 1.7 obtained by using CPC fiber tips in dummy sensors are maintained in in-vitro glucose sensor operation, as shown in Figure 4(b). This indicates that the CPC tipped fibers can be used just like plane-cut fiber tips for real-time glucose measurements, but with the advantage of an increased fluorescence signal and thus an increased signal-to-noise ratio of the sensor. This increase can directly enhance the detection limit whereas the sensitivity of the sensor will remain the same.

Alternatively the more efficient CPC tipped fiber can be used to reduce the optical power delivered by the light source in the sensors, if the current signal-to-noise ratio is sufficient. This reduction in optical power can reduce the sensor drift in continuous operation by reducing the photobleaching effect and thereby increase the sensor performance. The reduced power of the light source in the sensor will reduce the electrical power consumption and thereby increase the battery lifetime, reduce the battery size, thus further miniaturizing the portable reader unit for glucose sensing.

Our results have here demonstrated that the main reason for the observed difference between dummy sensor and real glucose sensor performance lies in the accurate bonding process of the assay compartment to the fiber, which gives slightly varying compartment lengths and thereby slightly different concentrations. However, this is just a matter of comparing two sensors working on the same concentration and is not a limitation on the operation of the CPC tipped sensor, which still can achieve the predicted optimum improvement in performance as compared to the plane-cut fiber sensor.

\section{CONCLUSION}

We made CPC tipped fiber based glucose sensors and characterized them for their increment in fluorescence pick-up efficiency in in-vitro glucose sensor operation. An increment factor of 1.7, as compared to the same sensor using a plane-cut fiber tip, was demonstrated in real sensor operation. Our results further demonstrate that the optimum increment factor, which is 3.0 in the particular fiber sensor design used here, can be achieved by optimizing the CPC fabrication process to repeatedly fabricate the ideal CPC shape.

\section{ACKNOWLEDGMENT}

The research leading to these results has received funding from the People Programme (Marie Curie Actions) of the European Union's Seventh Framework Programme FP7/20072013/ under REA grant agreement $n^{\circ} 608382$.

\section{REFERENCES}

[1] World Health Organization, "DiabetesFactSheet.", June 2016 http://www.who.int/mediacentre/factsheets/fs312/en/

[2] Newman, J.D., Turner, A.P.F., "Biosensors for Monitoring Glucose" in: Sensors Applications. J. Hesse, ed. (Wiley, 2008).

[3] K.-C. Liao, T. Hogen-Esch, F. J. Richmond, L. Marcu, W. Clifton, and G. E. Loeb, "Percutaneous fiber-optic sensor for chronic glucose monitoring in vivo," Biosens. Bioelectron., vol. 23, no.10, pp. $1458-$ 1465, May 2008.

[4] William L. Clarke., "The Original Clarke Error Grid Analysis (EGA)," Diabetes Technology \& Therapeutics, vol. 7, no. 5, pp. 776-779, Oct 2005.

[5] S. C. Warren-Smith, S. Heng, H. Ebendorff-Heidepriem, A. D. Abell, and T. M. Monro, "Fluorescence-Based Aluminum Ion Sensing Using a Surface-Functionalized Microstructured Optical Fiber," Langmuir, vol. 27, no. 9, pp. 5680-5685, April 2011.

[6] R. Ballerstadt, C. Evans, A. Gowda, and R. McNichols, "Fiber-coupled fluorescence affinity sensor for 3-day in vivo glucose sensing," J. diabetes Sci. Technol., vol. 1, no. 3, pp. 384-39, May 2007.

[7] J.C. Pickup, F. Khan, Z.L. Zhi, J. Coulter, D.J. Birch, "Fluorescence Intensity- and Lifetime-Based Glucose Sensing Using Glucose/Galactose-Binding Protein,” J. Diabetes Sci. Technol., vol. 7, no. 1, pp. 62-71, May 2013.

[8] J.B. Jensen, P.E. Hoiby, G. Emilianov, O. Bang, L.H. Pedersen, and A. Bjarklev, "Selective detection of antibodies in microstructured polymer optical fibers," Opt. Express, vol. 13, no. 15, pp. 5883-5889, July 2005.

[9] G. Emiliyanov, J.B. Jensen, O. Bang, P.E. Hoiby, L.H. Pedersen, E.M. Kjaer, and L. Lindvold, "Localized biosensing with Topas microstructured polymer optical fiber," Opt. Lett., vol. 32, no. 5, pp. 460-462, May 2007.

[10] G. Emiliyanov, P.E. Høiby, L.H. Pedersen, and O. Bang, "Selective serial multi-antibody biosensing with TOPAS microstructured polymer optical fibers," Sensors, vol. 13, no.3, pp.3242-325, Mar. 2013.

[11] S. Ko and S. A. Grant, "A novel FRET-based optical fiber biosensor for rapid detection of Salmonella typhimurium," Biosens. Bioelectron., vol. 21, no.7, pp. 1283 - 1290, Jan. 2006.

[12] N. Polley, S. Singh, A. Giri, P. K. Mondal, P. Lemmens, and S. K. Pal, "Ultrafast FRET at fiber tips: Potential applications in sensitive remote sensing of molecular interaction," Sens. Actuators B Chem., vol. 210, pp. 381 - 388, Apr. 2015

[13] K. E. Sapsford, L. Berti, and I. L. Medintz, "Materials for fluorescence resonance energy transfer analysis: Beyond traditional donor-acceptor combinations," Angew Chem Int Ed Engl., vol. 45, no. 28, pp. 45624588, July 2006.

[14] G. Chen, F. Song, X. Xiong, and X. Peng, "Fluorescent Nanosensors Based on Fluorescence Resonance Energy Transfer (FRET)," Ind. Eng. Chem. Res., vol. 52, no. 33, pp. 11228-11245, Jan. 2013.

[15] H.U. Hassan, K. Nielsen, S. Aasmul, O. Bang, "Polymer optical fiber compound parabolic concentrator tip for enhanced coupling efficiency 
for fluorescence based glucose sensors,” Biomed. Opt. Express, vol. 6, no. 12, pp. 5008-5020, Nov. 2015.

[16] C. Chen, Q. Xie, D. Yang, H. Xiao, Y. Fu, Y. Tan, S. Yao, "Recent advances in electrochemical glucose biosensors: a review", RSC Adv., vol. 3, no. 14, pp. 4473-4491, Dec. 2012.

[17] J. Wang, "Electrochemical glucose biosensors", Chem. Re., vol. 108, no. 2, pp. 57-69, Dec. 2007

[18] D.Sodzel et.al, "Continuous sensing of hydrogen peroxide and glucose via quenching of the UV and visible luminescence of $\mathrm{ZnO}$ nanoparticles," Microchim. Acta., vol. 182, no. 9, pp. 1819-1826, May 2015.

[19] I. a. Ges, F. Baudenbacher, "Enzyme electrodes to monitor glucose consumption of single cardiac myocytes in sub-nanoliter volumes," Biosens. Bioelectron., vol. 25, no. 5, pp. 1019-1024, Jan. 2010.

[20] E.-H. Yoo, S.-Y. Lee, "Glucose Biosensors: An Overview of Use in Clinical Practice," Sensors, vol. 10, no. 5, pp. 4558-4576, May 2010.

[21] A. Woehler, J. Wlodarczyk, and E. Neher, "Signal/noise analysis of FRET-based sensors," Biophys. J., vol. 99, no. 7, pp. 2344-2354, Oct. 2010.

[22] S. C. Warren-Smith, H- Ebendorff-Heidepriem, T.C. Foo, R. Moore, C. Davis, and T. M. Monro, "Exposed-core microstructured optical fibers for real-time fluorescence sensing", Opt. Express, vol. 17, no. 21, pp. $18533-18542$, Oct. 2009

[23] W. T. Welford and R. Winston, The Optics of Nonimaging Concentrators, Academic New York, 1978.

[24] L. Rindorf, O. Bang, "Sensitivity of photonic crystal fiber grating sensors: biosensing, refractive index, strain, and temperature sensing", J. Opt. Soc. Am. B., vol. 25, no. 3, pp. 310-324, Mar. 2008.

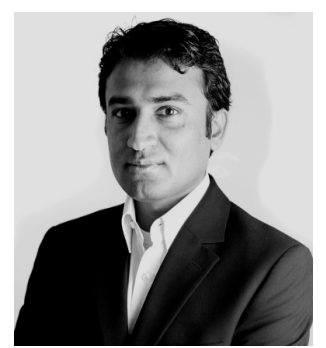

HAFEEZ UL HASSAN received the B.S. degree in Engineering Sciences from Ghulam Ishaq Khan Institute of Engineering Sciences \& Technology, Pakistan, in 2006, and the M.Sc degree in Photonics from Friedrich Schiller University, Jena, Germany in 2011. He is currently pursuing the Ph.D. degree in Photonic Engineering from Technical University of Denmark. His research interests are optical fiber and fiber sensors.

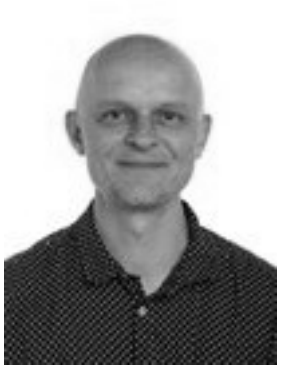

JAKOB JANTING received his MSc degree in Materials Science from the University of Aarhus, Denmark in 1991. Here he was employed as a researcher affiliated to the Tribology Department from 1991 to 1994 in an EU project where he was working on micro focus X-ray strain measurements. In 1994, he joined the Department for Sensors at Grundfos Research where he worked in a national project on materials for advanced MEMS sensor packaging. From 1998 to 2011 he was employed at DELTA, Danish Electronics, Light \& Acoustics, where focus of his work was on polymer microsystem encapsulation. In 2008 he was awarded the $\mathrm{PhD}$ degree for a thesis on reliability of polymer adhesive and coating materials for microsystem packaging. From 2011 to 2015 he was employed at Medtronic where he worked on reliable packaging of an on-body combined electrochemical and fiber optical microsensor device for continuous blood glucose monitoring and insulin delivery. In 2015 he got a Postdoc position in the Fiber Sensors and Supercontinuum group, The Department of Photonics Engineering, Technical University of Denmark where focus of his research is on microstructured Polymer Optical Fiber (mPOF) bio/chemical sensors.

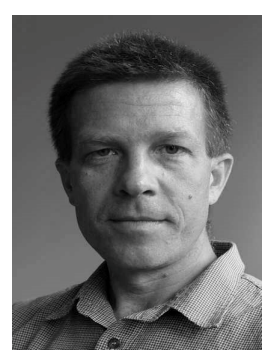

SOREN AASMUL is a Senior Principal R\&D Engineer in Medtronic Research and Technology Bakken Research Center $\mathrm{BV}$, Netherlands. He received his MSc in Electrical Engineering from Technical University of Denmark in 1992. Since then he has been working in the medical device business mainly in R\&D. His positions have ranged from early device research activities to development activities leading to $\mathrm{CE}$ marking and launch of a medical device.

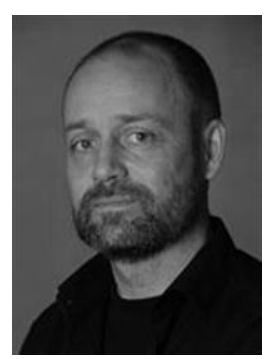

OLE BANG received the Master of Science degree in electrical engineering from the Technical University of Denmark in 1992 and the Doctor of Philosophy degree in nonlinear physics from the Technical University of Denmark, in 1993. From 1993 to 1995, he was a Postdoctoral Fellow at the Laboratoire de Physique, Ecole Normale Supérieure de Lyon, France, where he worked on discrete physical models of the nonlinear dynamics of biomolecules. From 1995 to 1999, he was a Research Fellow at the Optical Sciences Centre, Australian National University, Canberra, Australia, where he worked theoretically on nonlinear optics, in particular on solitons and modulational instability in materials with a quadratic nonlinearity and quasiphase-matching gratings. Since 1999, he has been an Associate Professor with the Technical University of Denmark, first at the Department of Informatics and Mathematical Modelling and, since 2003, with DTU Fotonik, Department of Photonics Engineering. His current research interests include microstructured optical fibers (MOFs) in silica and polymer, fiber-optical sensors for sensing biomolecules, stress, sound, and refractive index. He is also working on fabricating Bragg gratings, long-period gratings, and couplers in MOFs. Another main interest is supercontinuum generation and nonlinear fiber-optics in MOFs, as well as general nonlinear optics in nonlocal materials. Dr. Bang is a member of the Optical Society of America and also the Danish Optical Society. 